\title{
Thrombospondin-1 Plays an Essential Role in Yes-Associated Protein Nuclear Translocation during the Early Phase of Trypanosoma cruzi Infection in Heart Endothelial Cells
}

\author{
Ashutosh Arun ${ }^{1}$, Kayla J. Rayford ${ }^{1} \oplus$, Ayorinde Cooley ${ }^{1}$, Girish Rachakonda ${ }^{1}$, \\ Fernando Villalta ${ }^{1}$, Siddharth Pratap ${ }^{2}$, Maria F. Lima ${ }^{2,3}$, Nader Sheibani ${ }^{4}$ and \\ Pius N. Nde ${ }^{1, * \mathbb{D}}$ \\ 1 Department of Microbiology, Immunology and Physiology, Meharry Medical College, Nashville, \\ TN 37208, USA; aarun@mmc.edu (A.A.); krayford@mmc.edu (K.J.R.); acooley@mmc.edu (A.C.); \\ grachakonda@mmc.edu (G.R.); fvillalta@mmc.edu (F.V.) \\ 2 School of Graduate Studies and Research, Meharry Medical College, Nashville, TN 37208, USA; \\ spratap@mmc.edu (S.P.); mlima@med.cuny.edu (M.F.L.) \\ 3 Department of Molecular Cellular and Biomedical Sciences, School of Medicine, \\ The City College of New York, New York, NY 10031, USA \\ 4 Department of Ophthalmology and Visual Sciences, Biomedical Engineering and Cell and Regenerative \\ Biology, University of Wisconsin School of Medicine and Public Health, Madison, WI 53705, USA; \\ nsheibanikar@wisc.edu \\ * Correspondence: pnde@mmc.edu; Tel.: +1-615-327-6997
}

Received: 18 June 2020; Accepted: 9 July 2020; Published: 12 July 2020

Abstract: The protozoan parasite Trypanosoma cruzi is the causative agent of Chagas disease. This neglected tropical disease causes severe morbidity and mortality in endemic regions. About $30 \%$ of T. cruzi infected individuals will present with cardiac complications. Invasive trypomastigotes released from infected cells can be carried in the vascular endothelial system to infect neighboring and distant cells. During the process of cellular infection, the parasite induces host cells, to increase the levels of host thrombospondin-1 (TSP-1), to facilitate the process of infection. TSP-1 plays important roles in the functioning of vascular cells, including vascular endothelial cells with important implications in cardiovascular health. Many signal transduction pathways, including the yes-associated protein 1 (YAP)/transcriptional coactivator, with PDZ-binding motif (TAZ) signaling, which are upstream of TSP-1, have been linked to the pathophysiology of heart damage. The molecular mechanisms by which T. cruzi signals, and eventually infects, heart endothelial cells remain unknown. To evaluate the importance of TSP- 1 expression in heart endothelial cells during the process of $T$. cruzi infection, we exposed heart endothelial cells prepared from Wild Type and TSP-1 Knockout mouse to invasive T. cruzi trypomastigotes at multiple time points, and evaluated changes in the hippo signaling cascade using immunoblotting and immunofluorescence assays. We found that the parasite turned off the hippo signaling pathway in TSP-1KO heart endothelial cells. The levels of SAV1 and MOB1A increased to a maximum of $2.70 \pm 0.23$ and $5.74 \pm 1.45$-fold at 3 and $6 \mathrm{~h}$, respectively, in TSP-1KO mouse heart endothelial cells (MHEC), compared to WT MHEC, following a parasite challenge. This was accompanied by a significant continuous increase in the nuclear translocation of downstream effector molecule YAP, to a maximum mean nuclear fluorescence intensity of $10.14 \pm 0.40$ at $6 \mathrm{~h}$, compared to wild type cells. Furthermore, we found that increased nuclear translocated YAP significantly colocalized with the transcription co-activator molecule pan-TEAD, with a maximum Pearson's correlation coefficient of $0.51 \pm 0.06$ at $6 \mathrm{~h}$, compared to YAP-Pan-TEAD colocalization in the WT MHEC, which decreased significantly, with a minimum Pearson's correlation coefficient of $0.30 \pm 0.01$ at $6 \mathrm{~h}$. Our data indicate that, during the early phase of infection, upregulated TSP-1 is essential for the regulation of the hippo signaling pathway. These studies advance our understanding 
of the molecular interactions occurring between heart endothelial cells and T. cruzi, in the presence and absence of TSP-1, providing insights into processes linked to parasite dissemination and pathogenesis.

Keywords: Chagas heart disease; heart endothelial cells; hippo signaling; transcriptional enhancer factor (TEF) family/TEA domain (TEAD) family; Trypanosoma cruzi; yes-associated protein1

\section{Introduction}

Trypanosoma cruzi, the causative agent of Chagas disease, is an obligate intracellular hemoflagellate parasite that can infect all nucleated cells of the body. The disease, which was originally endemic in Mexico and other Latin American countries, is now present in all economically advanced countries of the world, including the United States, due to modern globalization making it a new global health threat [1-5]. Several modes of transmission including autochthonous transmission have been reported in the emerging infection regions of the world [6]. During the process of cellular infection, invasive T. cruzi trypomastigotes infected host cells and transform to replicative amastigotes within the infected cell. The amastigotes multiply and accumulate in the now distended cell, where they transform to invasive trypomastigotes, just before the cell burst, to release the invasive trypomastigotes. Some released trypomastigotes infect neighboring cells, while others are transported in blood through the host's vascular system to infect cells in other parts of the body. During transportation in the vascular system, invasive trypomastigotes interact with, and potentially infect, endothelial cells lining the internal surface of the vascular wall.

Matricellular proteins are extracellular matrix (ECM) proteins that interact with cells and other ECM components to regulate cellular behavior and ECM organization, but are not part of the structural elements of the ECM [7-10]. Thrombospondin-1 (TSP-1) is a complex homotrimeric secreted matricellular glycoprotein, and a member of the group A subfamily of five TSP family members [11]. The protein plays important roles in regulating several cellular processes through its molecular interactions with extracellular molecules, including matrix regulating enzymes, glycosaminoglycans, growth factors and diverse cellular receptors among others, thereby having an important role in tissue and cellular homeostasis [9,12-15].

In the vasculature, TSP-1 plays an important role in the function of vascular cells, including vascular smooth muscle cells, endothelial cells, fibroblasts and inflammatory cells, suggesting that the molecule has important implications in cardiovascular health [16]. Furthermore, we showed that, during the early phase of infection, the parasite induces the expression of TSP-1 in host cells, including primary human coronary artery smooth muscle cells to facilitate cellular infection [17]. We also showed that the expressed TSP- 1 interacts with T. cruzi calreticulin (TcCRT) on the surface of the parasite, to facilitate cellular infection, which was inhibited in the presence of the TcCRT monovalent Fab antibody [18]. Furthermore, an increase in the cellular expression of TSP-1 induced by the parasite is essential for dysregulating the levels of phosphorylated proteins and cellular signaling [19].

The Hippo signaling pathway, which was originally identified in Drosophila melanogaster, has now been extensively reported in mammalian cells. This pathway plays important evolutionarily conserved roles, in the regulation of organ size, cell proliferation, and apoptosis [20,21]. In mammals, when the canonical hippo pathway is turned on, the upstream mammalian sterile 20-like kinase 1/2 (MST1/2) is auto-phosphorylated, and it phosphorylates the regulatory protein SAV1. The complex phosphorylates the hydrophobic motifs of the large tumor suppressor kinase 1/2 (LATS1/2), which then phosphorylates the regulatory protein, MOB1. The phosphorylated and activated LATS1/2 then phosphorylate serine residues of hippo effector yes-associated protein (YAP), and the transcriptional coactivator with PDZ-binding motif (TAZ). The phosphorylation of YAP/TAZ is a signal for interaction with 14-3-3 for cytoplasmic retention or ubiquitination-mediated proteasomal and autolysosomal degradation [22-24]. Conversely, when the hippo pathway is turned off, MST1/2 are not phosphorylated, and they cannot 
signal the phosphorylation of downstream LATS1/2 and MOB1. Therefore, when YAP/TAZ are not phosphorylated, they can be translocated into the nucleus, where they interact with other TEA domain family members/transcription enhancer factor (TEADs/TEF) families of transcriptional co-activating factors, to promote the transcription of downstream genes, including TSP-1, which plays an important role in cell growth and proliferation [22]. Specifically, in a breast cancer model, it was suggested that, when the hippo signaling pathway is turned off, YAP-TEAD complex in the nuclear compartment directly induces the expression of TSP-1, which activates focal adhesion kinase, FAK [25]. The molecular mechanism by which T. cruzi signals and eventually infects endothelial cells remains to be elucidated. This knowledge will aid in the development of specific molecular intervention strategies.

We hypothesize that, during the early phase of molecular interaction between T. cruzi and heart endothelial cells, TSP-1 plays an important role in the dysregulation of the hippo signaling pathway. To delineate the significance of TSP-1 expression on hippo signaling during the early phase of infection, we challenged MHEC from wild type (WT) and TSP-1 knockout (TSP-1KO) mice with T. cruzi. We evaluated the kinetics of hippo signaling pathway, nuclear translocation of hippo effector molecule YAP, and its nuclear interaction with the co-activator protein TEAD. Here, we show that in the presence of TSP-1 (WT MHEC), the parasite induced a decrease in the unphosphorylated levels of SAV1 and MOB1A leading to a significant decrease in the nuclear translocation of YAP. This is accompanied by a decrease in nuclear co-localization of YAP and pan-TEAD. In the absence of TSP-1 (TSP-1 KO MHEC), the parasite induced an increase in the unphosphorylated levels of SAV1 and MOB1A, leading to an increase in the nuclear translocation of YAP. This increase in the nuclear translocation of YAP was accompanied by an increase in the nuclear co-localization of YAP and pan-TEAD.

\section{Results}

\subsection{Kinetics of the Levels of SAV1 and MOB1A in MHEC during the Early Phase of T. cruzi Infection}

To gain insight into the importance of TSP-1 expression in the regulation of hippo signaling effector proteins and their associated signaling cascades in MHEC during the early phase of T. cruzi infection, we challenged WT and TSP-1 KO MHEC with T. cruzi for different lengths of time (0, 1, 2, 3 and $6 \mathrm{~h}$ ), and analyzed the levels of unphosphorylated hippo signaling cascade proteins using immunoblot assays. We evaluated the levels of unphosphorylated SAV1 and MOB1A in WT and TSP-1KO MHEC at the different time points. We found that, when WT MHEC were challenged with T. cruzi, the levels of unphosphorylated SAV1 showed a general decrease trend that was very significant at $2 \mathrm{~h}(0.60 \pm 0.05)$, $p<0.001$ and at $3 \mathrm{~h}(0.64 \pm 0.07)-p<0.01$ compared to uninfected control (Figure $1 \mathrm{~A})$.

This was accompanied by a significant decrease in the unphosphorylated levels of MOB1A, which is downstream of SAV1. The unphosphorylated levels of MOB1A increased at one hour then significantly decreased at $2 \mathrm{~h}(0.61 \pm 0.08), p<0.001$ and at $3 \mathrm{~h}(0.52 \pm 0.07), p<0.01$, compared to control (Figure 1B).

When we challenged TSP-1KO MHEC (in the absence of TSP-1), with the parasite, we observed that the levels of unphosphorylated SAV1 showed a continuous significant increase, from $2 \mathrm{~h}(1.91 \pm 0.33)$, $p<0.01$ to $3 \mathrm{~h}(2.70 \pm 0.23), p<0.001$ before showing a non-significant increase at $6 \mathrm{~h}$ compared to control (Figure 1C). This was accompanied by an overall increase in the levels of unphosphorylated MOB1A, which was significantly increased at $1 \mathrm{~h}(2.28 \pm 0.40), p<0.05,3 \mathrm{~h}(1.48 \pm 0.21), p<0.05$, to a maximum of $(5.74 \pm 1.45), p<0.05$ at $6 \mathrm{~h}$ time point, compared to uninfected control (Figure 1D). These results indicate that the upstream Hippo signaling molecules are turned off in the absence of TSP-1 in MHEC challenged with T. cruzi. 
A
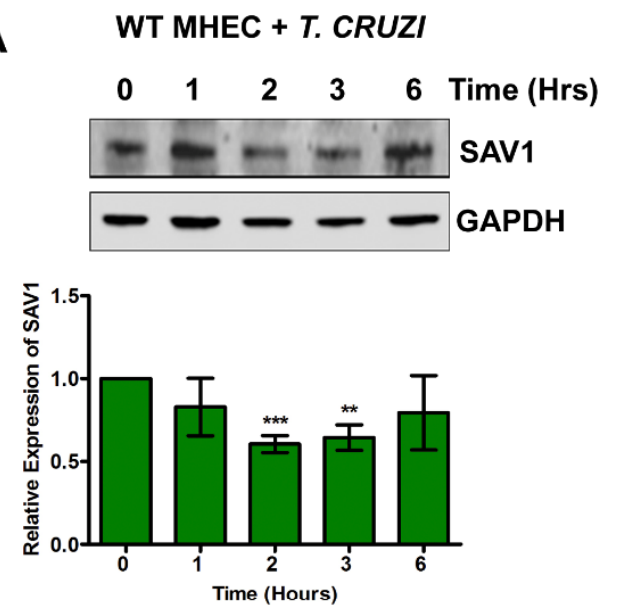

B
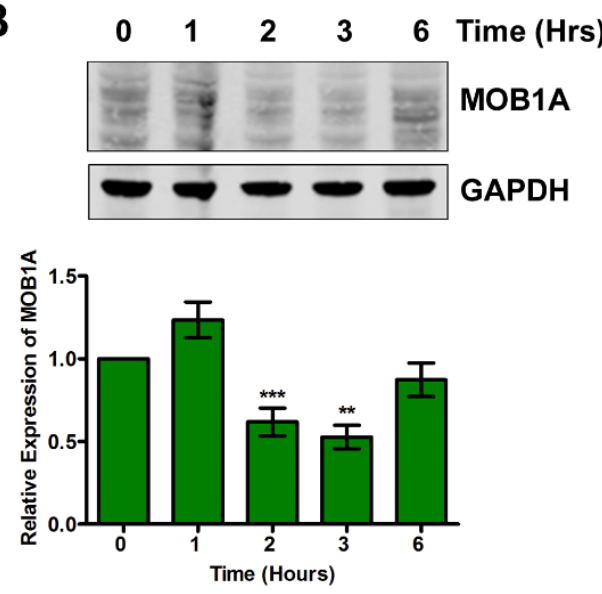

\section{TSP-1 KO MHEC + T. CRUZI}
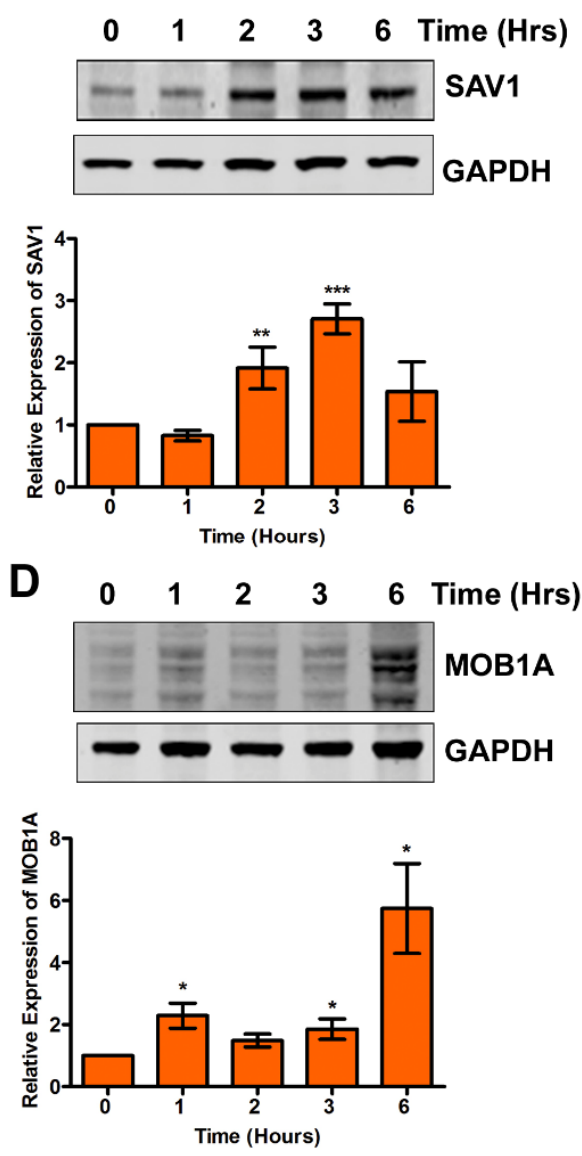

Figure 1. TSP-1 is essential for activation of hippo signaling cascade during the early phase of T. cruzi infection. Lysates $(20 \mu \mathrm{g})$ from WT or TSP-1KO MHEC challenged with T. cruzi at different time points were resolved by SDS-PAGE, blotted, and probed with antibodies against (A) SAV1, (B) MOB1A in WT MHEC and (C) SAV1, (D) MOB1A in TSP-1KO MHEC, and developed as described. The blots were stripped, reprobed with antibodies against housekeeping GAPDH and developed with the corresponding IRDye conjugated secondary antibody. The developed blots were scanned using the infrared fluorescence detection Odyssey Imaging Systems. The normalized fold change in the level of each unphosphorylated protein was determined and plotted in the bar graph for WT MHEC (A, lower panel) SAV1, (B, lower panel) MOB1A, respectively and for TSP-1KO MHEC (C, lower panel) SAV1 and (D, lower panel) MOB1A, respectively. The bar graphs represent mean values $\pm \mathrm{SE}$ from three independent biological replicates. The value of $p<0.05$ was considered significant. ${ }^{*} p<0.05$; ${ }^{* *}$ emphp $<0.01 ; * * * p<0.001$.

\subsection{Early T. cruzi Infection Increases Nuclear Translocation of YAP in TSP-1 KO MHEC}

Our data suggest that T. cruzi turns off the Hippo signaling pathway in the absence of TSP-1. When the hippo signaling is turned off, the downstream effector molecules are not phosphorylated, and therefore can be translocated into the nucleus. To evaluate if the increase in the levels of unphosphorylated upstream hippo signaling molecules correspond with an increase in nuclear translocation of downstream hippo effector molecule YAP, we challenged WT or TSP-1 KO MHEC with $T$. cruzi at different time points $(0,1,2,3$ and $6 \mathrm{~h})$, and analyzed the levels of nuclear translocation of downstream hippo effector molecule YAP, using confocal microscopy assays. We observed that when WT MHEC were challenged with T. cruzi trypomastigotes, the level of nuclear translocation of YAP significantly decreased with time, as shown by the nuclear intensity of the fluorophore in several fields. The mean nuclear intensity of YAP bound to the primary antibody detected by Alexa Fluor 
488 conjugated secondary antibody decreased from $1 \mathrm{~h}$, and the value became significant from $2 \mathrm{~h}$ (7.78 \pm 0.83$), p<0.01$, and continued to $3 \mathrm{~h}(8.16 \pm 0.01), p<0.01$ and finally $6 \mathrm{~h}(6.7 \pm 1.60), p<0.01$ compared with control (Figure 2A,B).

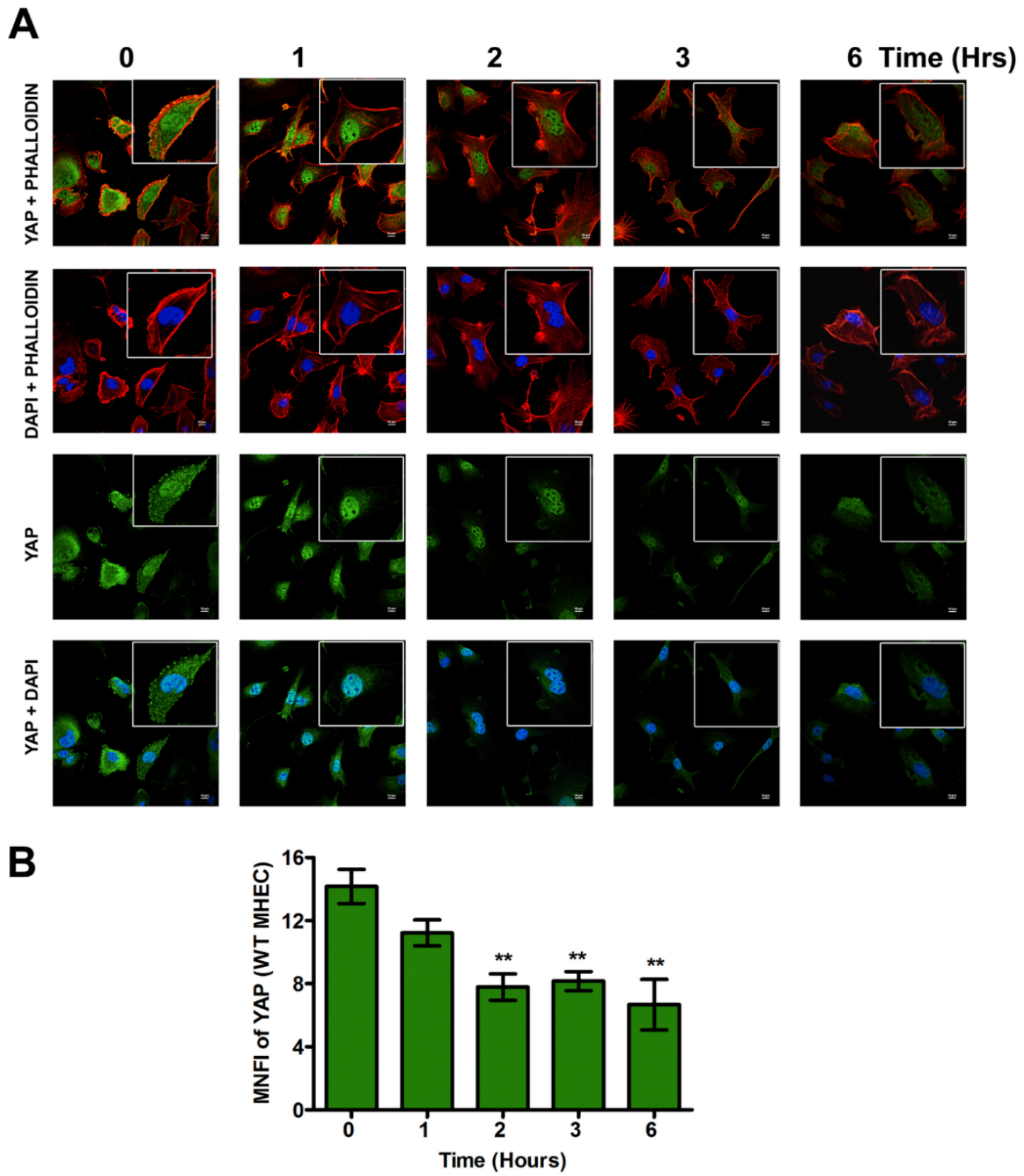

Figure 2. YAP is translocated into the nuclei of WT MHEC early during T. cruzi infection. WT MHEC grown on coverslips coated with $1 \%$ gelatin in 6-well culture plates were challenged with T. cruzi at different time points, washed, fixed, perforated with $0.1 \%$ Triton-X100, blocked with $3 \%$ BSA-PBS, and incubated at $4{ }^{\circ} \mathrm{C}$ overnight in solutions containing phalloidin and (A) mouse anti YAP antibodies. The cells were washed and reprobed with goat anti mouse Alexa Fluor 488 conjugated secondary antibody. The washed cells were mounted with mounting media containing DAPI to stain the nuclei. (B) Stained slides were analyzed by confocal microscopy and the mean nuclear fluorescence intensity (MNFI) values were plotted for YAP. Images were captured at $60 \times$ at scale bar $10 \mu \mathrm{m}$. Each confocal microscopy image is a representative of three independent biological replicates. The bar graphs represent MNFI values \pm SE from three independent biological replicates. The value of $p<0.05$ was considered significant. ${ }^{* *} p<0.01$. 
Conversely, when we challenged TSP-1KO MHEC with the parasite, we observed that the nuclear intensity of YAP translocated into the nucleus showed a gradual continuous significant increase from $2 \mathrm{~h}(7.17 \pm 1.30), p<0.01$ to $3 \mathrm{~h}(10.14 \pm 0.40), p<0.01$, and finally to a maximum at $6 \mathrm{~h}(11.68 \pm 2.12)$, $p<0.01$ compared to control (Figure 3A,B).

A
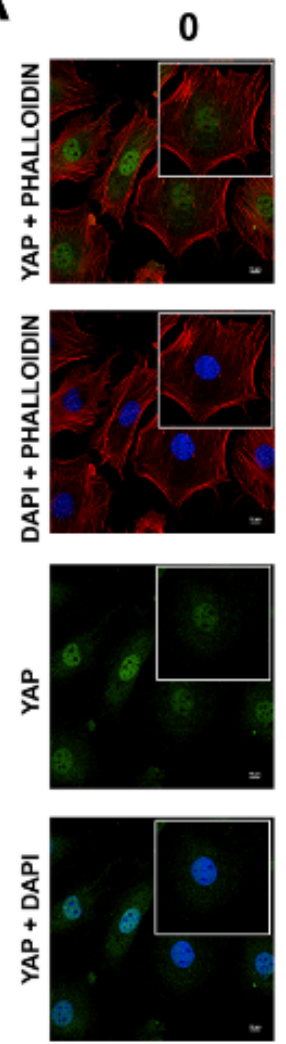

B
1
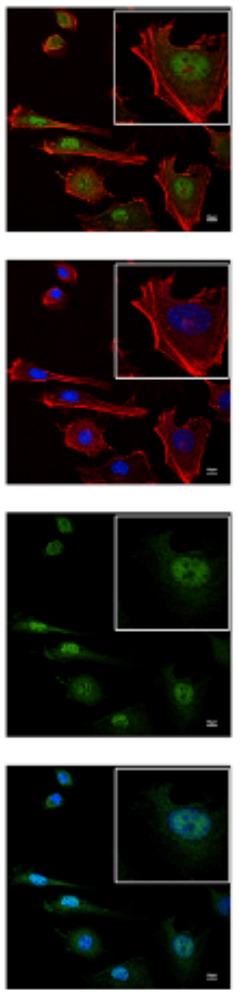

2
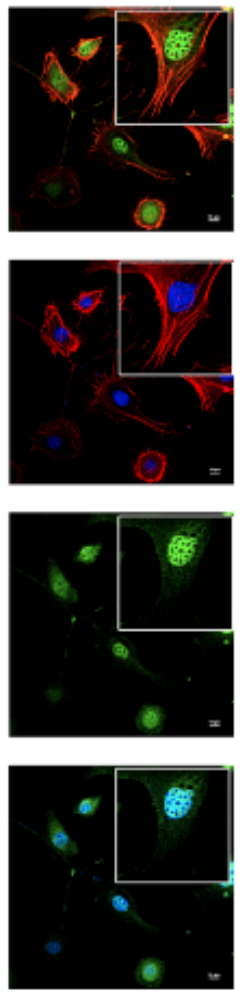

3
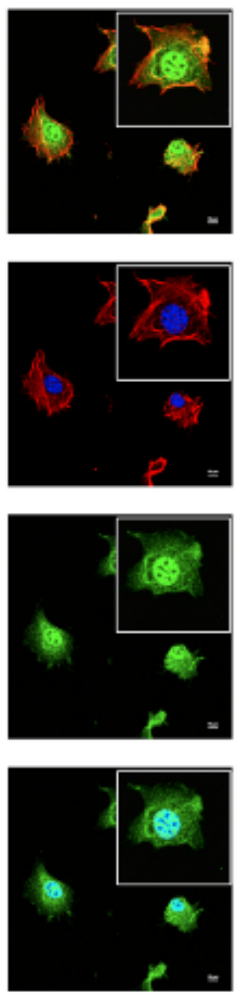

6 Time (Hrs)
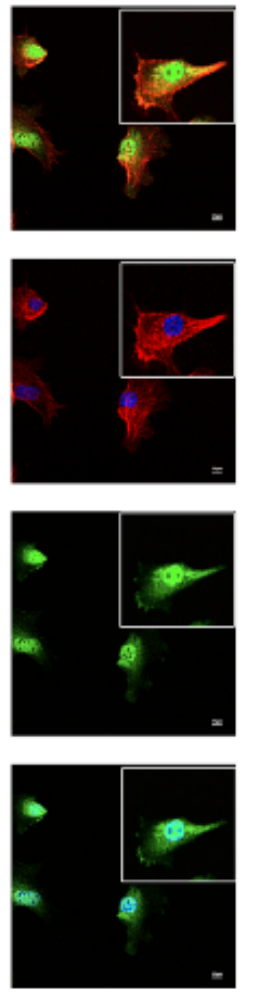

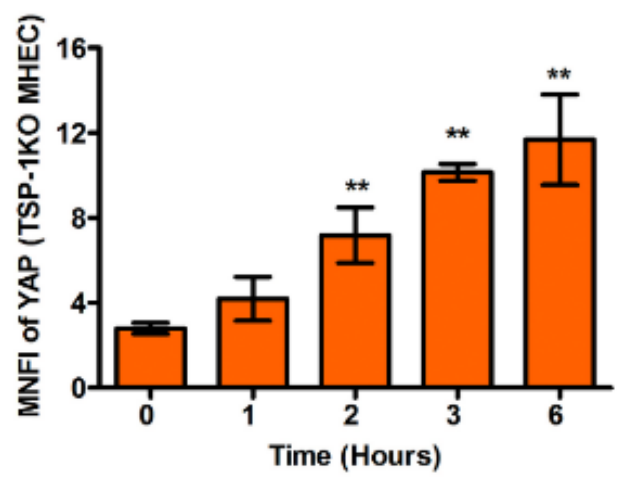

Figure 3. T. cruzi induces significant translocation of YAP into the nuclei of TSP-1KO MHEC early during T. cruzi infection. TSP-1KO MHEC grown on coverslips coated with $1 \%$ gelatin in 6 well culture plates were challenged with T. cruzi at different time points, washed, fixed, perforated with $0.1 \%$ Triton-X100, blocked with $3 \%$ BSA-PBS, and incubated at $4{ }^{\circ} \mathrm{C}$ overnight, in solutions containing phalloidin and (A) mouse anti YAP antibodies. The cells were washed and reprobed with goat anti mouse Alexa Fluor 488 conjugated secondary antibody. The washed cells were mounted with mounting media containing DAPI to stain the nuclei. (B) Stained slides were analyzed by confocal microscopy and the mean nuclear fluorescence intensity (MNFI) values were plotted for YAP. Images were captured at $60 \times$ at scale bar $10 \mu \mathrm{m}$. Each confocal microscopy image is a representative of three independent biological replicates. The bar graphs represent MNFI values \pm SE from three independent biological replicates. The value of $p<0.05$ was considered to be significant. ${ }^{* *} p<0.01$. 
A comparison of the differences in the nuclear translocation of YAP showed that in the presence of TSP-1, there is a significant continuous decrease in YAP nuclear translocation with time, compared to the absence of TSP- 1 where we observe a significance continuous increase in YAP nuclear intensity. At $6 \mathrm{~h}$ post infection, WT MHEC had a higher number of parasites per cell $(4.2 \pm 0.15)$ than TSP-1 KO MHEC $(0.12 \pm 0.02)$.

\subsection{YAP and Pan-TEAD Co-Localize in the Nucleus of MHEC during the Early Phase of T. cruzi Infection}

The downstream hippo effector molecule YAP is a transcription factor that interacts with its co-activator TEAD to bind to DNA. To evaluate whether the YAP translocated to the nucleus interacts with TEAD, MHEC (WT and TSP-1 KO) were challenged with T. cruzi (10 parasites per cell), then the cells were harvested or fixed after different time points after infection $(0,1,2,3$ and $6 \mathrm{~h})$. Then, they were analyzed for the levels of nuclear co-localization of the downstream effector molecule YAP with TEAD, by measuring the Pearson's correlation of both proteins in the nucleus using confocal microscopy assays. In the presence of TSP- 1 in WT MHEC, we observed that the mean Pearson's correlation of YAP and pan-TEAD nuclear colocalization decreased gradually with time, and was significant at $6 \mathrm{~h}$ $(0.30 \pm 0.01), p<0.05$, compared to control (Figure $4 \mathrm{~A}, \mathrm{~B}$ ).

In the absence of TSP-1, we observed a gradual increase in the nuclear colocalization of YAP and pan-TEAD, as shown by a similar increase in mean Pearson's correlation coefficient to a maximum at $6 \mathrm{~h}(0.51 \pm 0.06), p<0.05$, compared to uninfected control (Figure 5A,B).

A comparison of the nuclear colocalization of YAP and pan-TEAD showed a gradual decrease in WT MHEC compared to a gradual increase in the absence of TSP-1 in MHEC TSP-1KO, all of which were significant at $6 \mathrm{~h}$ time point, compared to control. 
A
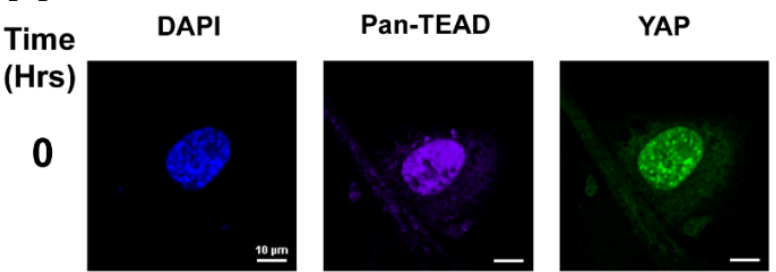

YAP + Pan-TEAD Merged with DAPI
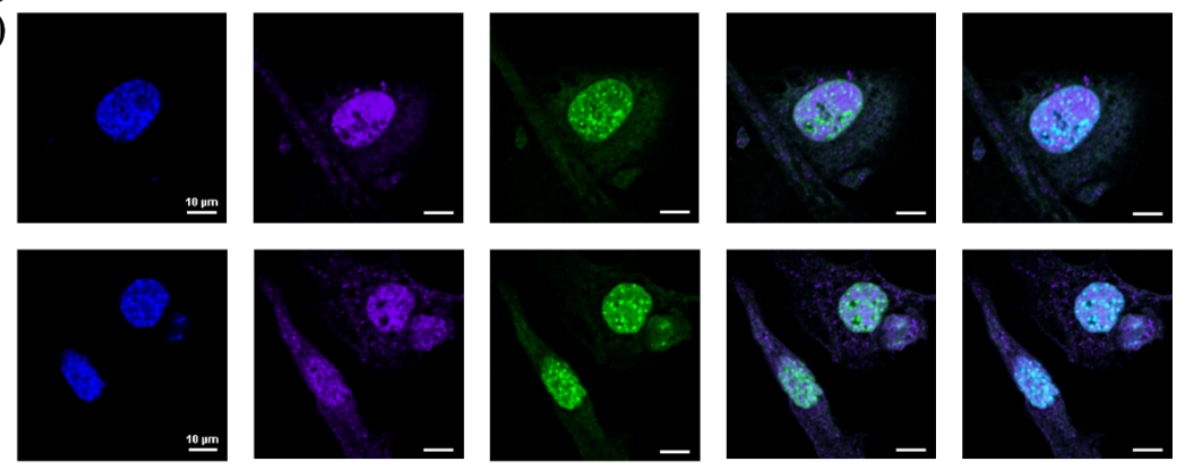

2
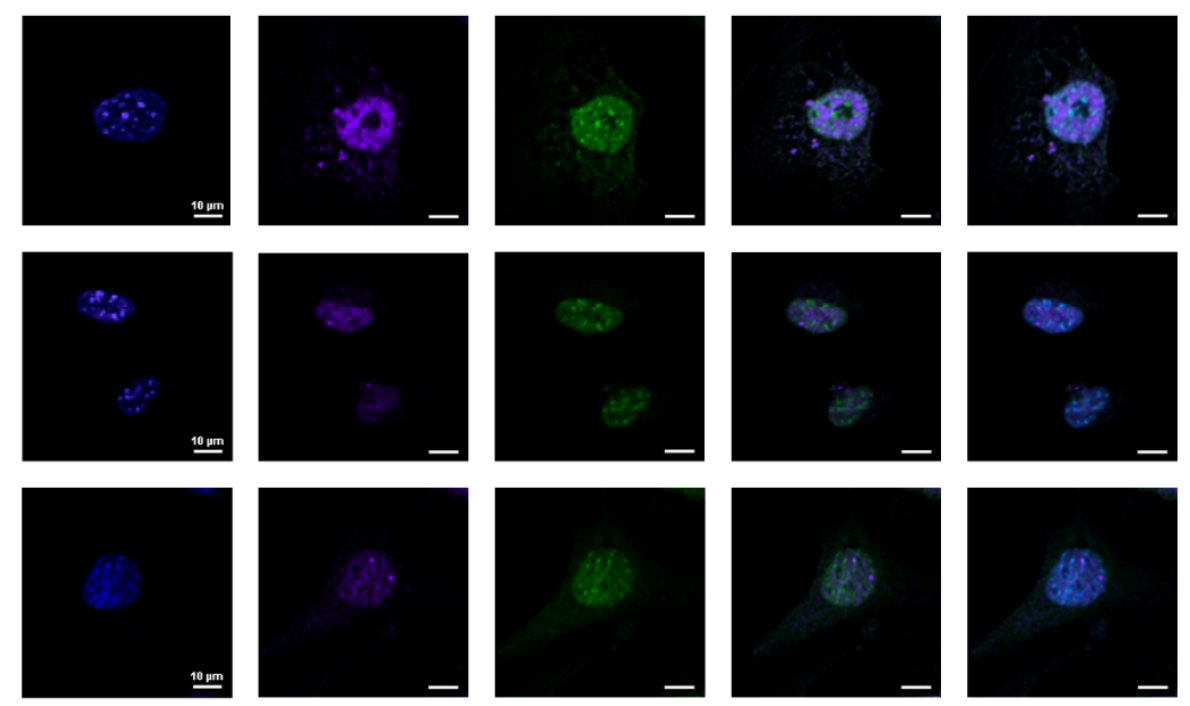

B

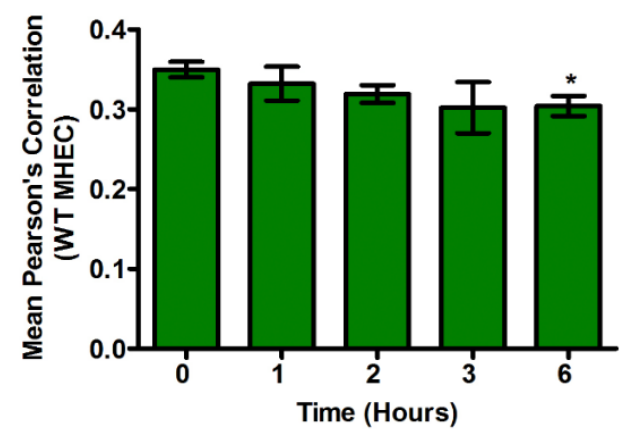

Figure 4. YAP and pan-TEAD are colocalized in the nuclei of WT MHEC during the early phase T. cruzi infection. WT MHEC grown on coverslips coated with $1 \%$ gelatin in 6 well culture plates were challenged with T. cruzi at different time points, washed, fixed, perforated with $0.1 \%$ Triton-X100, blocked with $3 \%$ BSA-PBS, and incubated at $4{ }^{\circ} \mathrm{C}$ overnight, in solutions containing phalloidin and (A) mouse anti YAP and rabbit anti pan-TEAD antibodies. The washed cells were reprobed with a cocktail of goat anti mouse Alexa Fluor 488 and goat anti rabbit Alexa Fluor 647 conjugated secondary antibodies. The cells were washed and mounted with mounting media containing DAPI to stain the nuclei. Stained slides were analyzed by confocal microscopy and images were captured at $60 \times$ at scale bar $10 \mu \mathrm{m}$. The mean fluorescence intensities of the merged signals were analyzed using confocal microscopy software to generate Pearson's correlation coefficients. (B) The bar graphs represent Pearson's correlation coefficients values \pm SE from three independent biological replicates. Each confocal microscopy image is a representative of three independent biological replicates. The value of $p<0.05$ was considered significant. ${ }^{*} p<0.05$. 
A
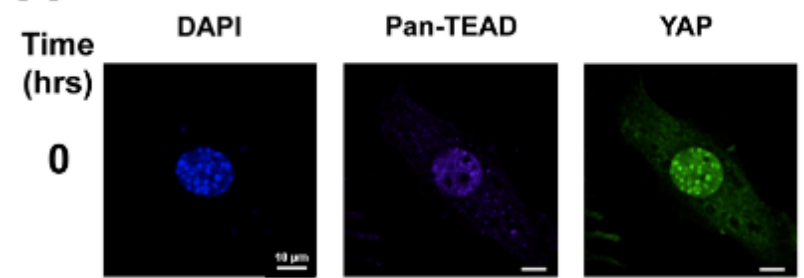

YAP + Pan-TEAD Merged with DAPI

1
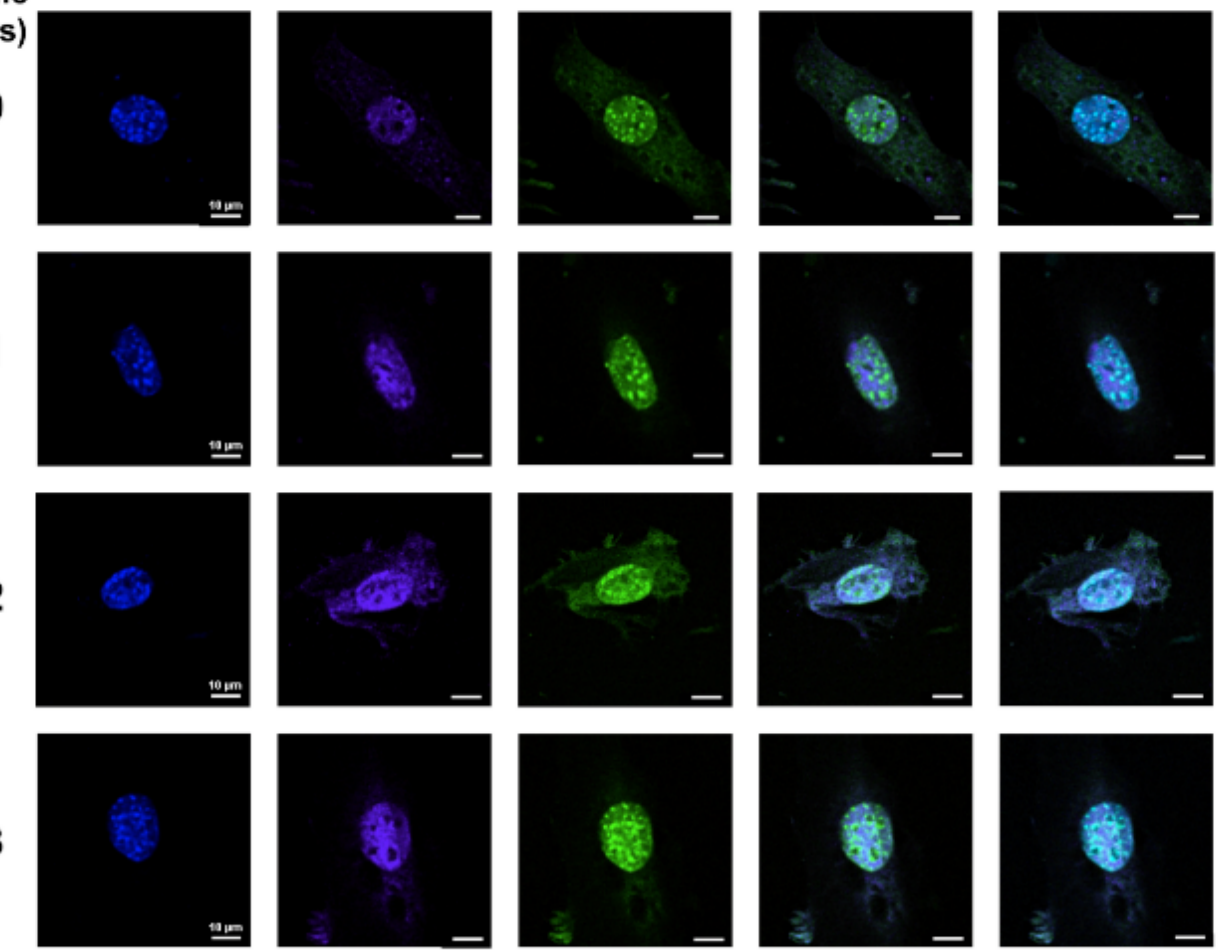

6
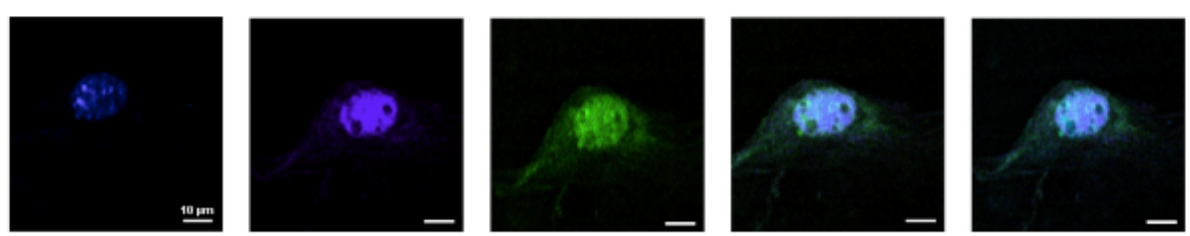

B

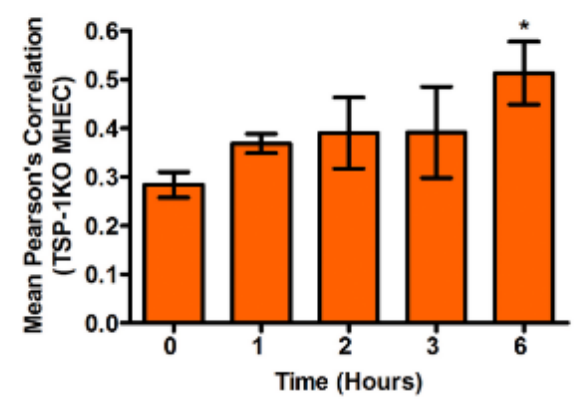

Figure 5. Transcriptional co-activators YAP and pan-TEAD are colocalized in the nuclei of TSP-1KO MHEC during the early phase of T. cruzi infection. TSP-1KO MHEC grown on coverslips coated with $1 \%$ gelatin in 6 well culture plates were challenged with T. cruzi at different time points, washed, fixed, perforated with $0.1 \%$ Triton-X100, blocked with $3 \%$ BSA-PBS, and incubated at $4{ }^{\circ} \mathrm{C}$ overnight in solutions containing phalloidin, and the following antibodies; (A) mouse anti YAP and rabbit anti pan-TEAD antibodies. The cells were washed and reprobed with a cocktail of goat anti mouse Alexa Fluor 488 and goat anti rabbit Alexa Fluor 647 conjugated secondary antibodies. The cells were washed and mounted with mounting media containing DAPI to stain the nuclei. Stained slides were analyzed by confocal microscopy and images were captured at $60 \times$ at scale bar $10 \mu \mathrm{m}$. Each confocal microscopy image is a representative of three independent biological replicates. The mean fluorescence intensities of the merged signals were analyzed using confocal microscopy software to generate Pearson's correlation coefficients. (B) The bar graphs represent Pearson's correlation coefficients values \pm SE from three independent biological replicates. The value of $p<0.05$ was considered to be significant. ${ }^{*} p<0.05$. 


\section{Discussion}

Chagas disease is a major pathology associated with severe morbidity and mortality in T. cruzi-infected patients. The molecular mechanisms through which the parasite causes heart disease remains to be clearly elucidated despite ongoing research. Cardiac pathology caused by T. cruzi infection includes the general cardiac enlargement of all four chambers-myocarditis, vasculitis, and vascular dilation, among others [26]. The parasite can infect all nucleated cells of the heart, including myocytes, fibroblasts, neurons, adipocytes and endothelial cells $[2,27,28]$. Invasive trypomastigotes carried in the blood will interact with and infect heart endothelial cells causing disease. However, the molecular mechanisms involved remain undefined.

We showed previously that the parasite upregulates host TSP-1 during the early phase of infection and the knock down of host TSP-1 by RNAi reduces the level of cellular infection by T. cruzi [17]. Furthermore, we also showed that the parasite interacts with host TSP-1 through T. cruzi calreticulin (TcCRT) located on the surface of the parasite [18]. The preincubation of host cells with an inhibitor of janus kinase (JNK) which is upstream of TSP-1, reduced the upregulation of TSP-1 induced by the parasite during the early phase of infection, indicating that TSP-1 upregulation is important and plays an essential role during infection [19]. The host cell signaling mechanism(s) regulated by the expressed host TSP-1 to facilitate infection and T. cruzi mediated pathogenesis remains to be elucidated. Elucidating the roles that host TSP-1 plays during the early phase of cellular infection, including the dysregulation of host cell signaling responses, will enhance our understanding of T. cruzi pathogenesis.

Although TSP-1 is one of the downstream genes of the hippo signaling pathway, which has been reported to play a role in organ enlargement and fibrogenic responses, the role that it may play in the regulation of hippo signaling pathway in heart endothelial cells during the early phase of T. cruzi infection is unknown $[14,20,21,25]$. Therefore, we hypothesize that $T$. cruzi trypomastigotes will dysregulate the hippo signaling pathway in heart endothelial cells during the early phase of cellular infection in a TSP-1 dependent manner. To elucidate the significance of TSP-1 in hippo signaling during the early phase of cellular infection of heart endothelial cells, we challenged MHEC from WT and TSP-1KO mice with invasive T. cruzi trypomastigotes, for various lengths of time $(0,1,2,3$ and $6 \mathrm{~h})$, and evaluated whether the hippo signaling pathway was turned on or off in the presence or absence of TSP-1. Our results show that the parasite turned "off" the hippo signaling pathway of MHEC in a TSP-1 dependent manner. Specifically, in the absence of TSP-1, the levels of unphosphorylated SAV1 and MOB1A proteins, which play an important role in hippo signaling [29,30], were significantly increased (Figure 1C,D). Our findings agree with others who suggested that the levels of unphosphorylated YAP is increased when SAV1 and MOB1A are not phosphorylated [31]. In the absence of TSP-1 in TSP-1 KO MHEC, the level of YAP significantly increased with time. The parasite turned "on" the hippo signaling pathway cascade proteins of WT MHEC, which expresses TSP-1 (Figure 1A,B). Furthermore, we showed that the levels of the transcriptional co-activator, $\mathrm{YAP}$, translocated in to the nucleus significantly increased with time to a maximum at $6 \mathrm{~h}$ in the TSP-1 KO MHEC (Figure 3A,B). Our data agree with reports in the literature, suggesting that when the hippo signaling pathway is turned off, the downstream effector transcriptional co-activator molecule YAP is translocated into the nucleus [32,33]. Meanwhile, in the presence of TSP-1, as in WT MHEC, the hippo signaling pathway is turned on, and the levels of transcriptional co-activator translocated to the nucleus significantly decrease with time (Figure 2A,B). Furthermore, in agreement with the upstream observations, our data showed that nuclear translocated YAP colocalized with its transcriptional co-activator TEAD. An increase in the levels of nuclear translocated YAP leads to an increase in its nuclear colocalization with pan-TEAD, in the absence of TSP-1 (Figure 5A,B), compared to a decrease in the nuclear YAP-pan-TEAD colocalization in WT MHEC that expresses TSP-1 (Figure 4A,B). Our observations support the current literature, which suggests that YAP interacts with its transcriptional co-activator pan-TEAD in the nuclear compartment. These results show, for the first time, that T. cruzi infection enhances nuclear translocation of YAP and the colocalization of YAP with pan-TEAD in the nuclei of TSP-1 KO MHEC compared to WT MHEC. 
We showed that T. cruzi upregulates the levels of TSP-1 during the process of infection [19,34]. Others showed that TSP-1 is one of the downstream molecules of the hippo signaling cascade [22,25]. This suggests that, in the absence of TSP-1, the parasites turned off the hippo signaling pathway, in an attempt to increase the level of host TSP-1, which is important in infection. Additionally, it was suggested that the level of TGF- $\beta$ increases during the process of T. cruzi infection, and TGF- $\beta$ inhibitor therapeutic use decreased fibrogenic responses during T. cruzi infection [35-37]. Others have suggested that TSP-1 can activate TGF- $\beta$, a profibrotic cytokine [38-40]. Taken together, this further supports our concept that an increase in the level of TSP-1 induced by the parasite is essential for T. cruzi infection and pathogenesis.

\section{Materials and Methods}

\subsection{Ethics Statement}

The animal study was carried out in accordance with the protocol number 150514PN093, approved by the Institutional Animal Care and Use Committee (IACUC) of Meharry Medical College.

\subsection{Generation and Culture of Mouse Heart Endothelia Cells (MHEC)}

MHEC were generated and maintained, as previously described for retinal EC [41]. Briefly, hearts were harvested from 4-week-old wild type or TSP-1KO Immorto mice, as detailed [41]. Hearts (from 3-4 mice) were pooled together, rinsed with plane DMEM (non-supplemented), minced into small pieces in a $60 \mathrm{~mm}$ tissue culture dish using sterilized razor blades, and digested in $5 \mathrm{~mL}$ of collagenase type I ( $1 \mathrm{mg} / \mathrm{mL}$ in serum free DMEM, Worthington, Lakewood, NJ, USA), for $30-45 \mathrm{~min}$ at $37{ }^{\circ} \mathrm{C}$. Following digestion, DMEM with $10 \%$ FBS was added, and cells were pelleted. The cellular digests were then filtered through a double layer of sterile $40 \mu \mathrm{m}$ nylon mesh (Sefar America Inc., Fisher Scientific, Hanover Park, IL, USA), and centrifuged at 400 $\mathrm{g}$ for $10 \mathrm{~min}$ to pellet cells. Cells were washed twice with DMEM containing $10 \%$ FBS, resuspended in $1.5 \mathrm{~mL}$ medium (DMEM with 10\% FBS), and incubated with sheep anti-rat magnetic beads, pre-coated with anti-PECAM-1 as described [41]. After affinity binding, magnetic beads were washed six times with DMEM with 10\% FBS and bound cells in endothelial cell growth medium were plated into a single well of a 24 well plate, pre-coated with $2 \mu \mathrm{g} / \mathrm{mL}$ of human fibronectin (BD Biosciences, Bedford, MA, USA). Endothelial cells were grown in DMEM containing 10\% FBS, $2 \mathrm{mM}$ L-glutamine, $2 \mathrm{mM}$ sodium pyruvate, $20 \mathrm{mM}$ HEPES, $1 \%$ non-essential amino acids, $100 \mu \mathrm{g} / \mathrm{mL}$ streptomycin, $100 \mathrm{U} / \mathrm{mL}$ penicillin, freshly added heparin at $55 \mathrm{U} / \mathrm{mL}$ (Sigma, St. Louis, MO, USA), endothelial growth supplement $100 \mu \mathrm{g} / \mathrm{mL}$ (Sigma, St. Louis, MO, USA), and murine recombinant interferon- $\gamma$ (R \& D, Minneapolis, $\mathrm{MN}$, USA), at 44 units $/ \mathrm{mL}$. Cells were maintained at $33{ }^{\circ} \mathrm{C}$ with $5 \% \mathrm{CO}_{2}$. Cells were progressively passed to larger plates, maintained, and propagated in $1 \%$ gelatin-coated $60 \mathrm{~mm}$ dishes. To confirm that these cells are EC, we examined the expression of two endothelial cell specific markers, PECAM- 1 and VE-cadherin, by FACS analysis. Nearly $100 \%$ of wild type and TSP1 ${ }^{-/}$MHEC expressed high levels of these markers on their surface (not shown). Early passage cultures of MHEC wild type (WT MHEC) and TSP-1KO (TSP-1KO MHEC) were maintained in growth medium and used for the studies outlined here.

\subsection{Rat Heart Myoblast Culture}

Rat heart myoblasts (RHM) were maintained in DMEM containing glutamax (Life Technologies, Grand Island, NY, USA), 10\% heat inactivated fetal bovine serum (Life Technologies), $1 \%$ penicillin/streptomycin (Life Technologies) and $1 \%$ non-essential amino acid and multivitamin (Life Technologies). RHM were grown in a humidified tissue culture incubator at $37^{\circ} \mathrm{C}$ and $5 \% \mathrm{CO}_{2}$.

\subsection{T. cruzi Trypomastigote Culture and Infection Assays}

RHM monolayers were cultured in complete media to $80 \%$ confluence. The cells were infected with T. cruzi trypomastigotes clone MMC 20A (Tulahuen strain). Infected heart myoblast monolayers 
were fed with fresh complete media (supplemented media) daily. A pure population of highly invasive T. cruzi trypomastigotes released in cell culture supernatants were harvested as previously described [42-44]. The parasites were washed in Hanks' balanced salt solution (HBSS) and resuspended in MHEC growth medium without supplement, at a working concentration of $1 \times 10^{7}$ parasites $/ \mathrm{mL}$, for use in subsequent experiments. For infection assays, approximately $85 \%$ confluent MHEC (WT or TSP-1KO) monolayers were starved in medium without supplements, followed by the addition of invasive T. cruzi trypomastigotes, at a ratio of 10 parasites per cell. The cells challenged with the parasites were incubated for different time points-1, 2, 3 and $6 \mathrm{~h}$. Parasites were washed off with $1 \times$ DPBS (without calcium/magnesium), and the cells were either processed immediately, or stored at $-80{ }^{\circ} \mathrm{C}$ for western blot analysis. Each time point was done using three independent T75 flask, for use in the western blotting experiments, and three independent sets of 6 well culture plates containing coverslip coated with $1 \%$ gelatin, for use in confocal immunofluorescence microscopy assays. Mock-infected (medium only) MHECs were used as controls for each time point.

\subsection{Immunoblotting Assays}

Uninfected control and parasite challenged cell monolayers were lysed in RIPA buffer (Life Technologies), containing protease inhibitor cocktail set III at 1:100, (Calbiochem, Gibbstown, NJ, USA) and phosphatase inhibitor cocktails 2 and 3, at 1:100 each (Sigma Aldrich, St. Louis, MO, USA). Whole cell lysates $(20 \mu \mathrm{g} /$ well) were separated by SDS-PAGE on a $4-15 \%$ gradient polyacrylamide gels, and transferred onto nitrocellulose membranes (Life Technologies). The membranes were incubated in Intercept TBS Blocking Buffer (LI-COR Biosciences, Lincoln, Nebraska, USA), followed by incubation with an appropriate primary antibody diluted at 1:1000 at $4{ }^{\circ} \mathrm{C}$ overnight on a shaker; mouse anti-SAV1 monoclonal antibody (Santa Cruz Biotechnology, Dallas, TX, USA; cat. no. sc-374366), mouse anti-GAPDH monoclonal antibody (Santa Cruz Biotechnology, cat. no. sc-47724), mouse anti-MOB1A monoclonal antibody (Life Technologies, catalog. no. MA5-31801). The blots were washed and incubated with the corresponding IRDye secondary antibody (LI-COR $800 \mathrm{CW}$ or $680 \mathrm{RD}$ anti-mouse/anti-rabbit) in blocking buffer containing $0.01 \%$ Tween 20 for $1 \mathrm{~h}$ at room temperature. The blots were washed and scanned using the infrared fluorescence detection Odyssey Imaging Systems (LI-COR Biosciences) to visualize the bound antibody. Housekeeping GAPDH signal was used for normalization of loading differences. Each experiment was done in triplicate, and the quantitation of band intensity was performed by densitometry using Image J. A statistical analysis of each parameter for the T. cruzi treated groups was compared with non-treated control groups (0 Hours) using student's $t$-test or one-way ANOVA (non-parametric), with Newman-Keuls post-hoc test. The difference was considered statistically significant if ${ }^{*} p<0.05$, ${ }^{* *} p<0.01$ and ${ }^{* * *} p<0.001$ vs. control.

\subsection{Immunofluorescence Assays}

MHECs (WT or TSP-1KO) were seeded on coverslip coated with $1 \%$ gelatin in 6 well culture plate. T. cruzi trypomastigotes Tulahuen strain clone MMC 20A (10 parasites per cell) were incubated with the MHEC for different time points-1, 2,3 and $6 \mathrm{~h}$. The parasites were washed off with DPBS. The cells were fixed with $4 \%$ paraformaldehyde for $5 \mathrm{~min}$ at room temperature and washed with 1X DPBS. Fixed cells were perforated with $0.1 \%$ Triton-X100 in TBS for 5 min, and blocked with $3 \%$ BSA-PBS for $30 \mathrm{~min}$ at room temperature. For an evaluation of nuclear translocation, slides were incubated with mouse anti-YAP monoclonal antibody diluted 1:100 (Santa Cruz Biotechnology, cat. no. sc-271134) and phalloidin (1:2000) in $1 \%$ BSA-PBS at $4{ }^{\circ} \mathrm{C}$ overnight. The cells were washed with $1 \%$ BSA-PBS and re-probed with goat anti mouse IgG secondary antibody conjugated with Alexa Fluor 488 (1:1000) in wash buffer for $1 \mathrm{~h}$. They were further washed and mounted with mounting media containing DAPI (Life Technologies) to stain the nuclei. For colocalization assays, the fixed, perforated and blocked cells on the slides were incubated with a mixture of antibodies, containing mouse anti-YAP monoclonal antibody diluted 1:100 (Santa Cruz Biotechnology cat no. sc-271134), rabbit anti-pan-TEAD (D3F7L) 
monoclonal antibody (Cell signaling Technology, cat no.13295) diluted 1:100, and phalloidin (1:2000) at $40{ }^{\circ} \mathrm{C}$ overnight. The cells were washed and the bound primary antibody were detected using the corresponding secondary antibody cocktails; goat anti-rabbit Alexa Fluor 647 and goat anti-mouse Alexa Fluor 488 antibodies, diluted 1:1000 in 1\% BSA-PBS, for $1 \mathrm{~h}$ at room temperature, washed and mounted with mounting media containing DAPI to stain the nuclei. The coverslips with treated cells were transferred on to glass slides and sealed. Stained slides were analyzed using the Nikon A1R confocal microscope, located at the Centralized Core Facility at Meharry Medical College. Cellular and nuclear intensities, including Pearson's correlation coefficient for the colocalization of signals were determined by imaging software NIS Elements AR Analysis version 5.20.02 64-bit.

\subsection{Statistical Analysis}

Data from at least three independent experiments are expressed as mean \pm SEM. Statistical comparisons were made between controls and T. cruzi treated groups. Statistical comparisons were analyzed using Student's $t$-test or a one-way analysis of variance (ANOVA) for multiple groups of data, followed by a Newman-Keuls test. $p$-value strength increases with number of asterisks $p<0.05\left(^{*}\right), p<0.01\left({ }^{* *}\right)$, and $p<0.001\left(^{* * *}\right)$. Statistical analyses were done using GraphPad Prism (GraphPad Software, San Diego, CA, USA).

Author Contributions: Conceptualization, P.N.N.; methodology, A.A. and P.N.N.; validation, A.A., K.J.R. and G.R.; formal analysis, A.A., K.J.R and A.C.; investigation, A.A. and K.J.R. resources, F.V., M.F.L. and N.S.; data curation, A.A. and P.N.N.; writing-review and editing, A.A., K.J.R., A.C., G.R. and S.P.; visualization, A.A., K.J.R., G.R., A.C. and S.P.; supervision, P.N.N.; project administration, P.N.N.; funding acquisition, F.V., M.F.L., N.S. and P.N.N. All authors have read and agreed to the published version of the manuscript.

Funding: This research was funded by NIH grants 1SC1AI127352, 5R25GM059994, and U54MD007586. The NS lab is supported by an unrestricted award from Research to Prevent Blindness to the Department of Ophthalmology and Visual Sciences, Retina Research Foundation, NIH grants P30 EY016665, P30 CA014520, and R01 EY026078. NS is a recipient of a RPB Stein Innovation Award.

Acknowledgments: We are grateful to the Molecular Biology Core Facility and the Morphology Core at Meharry Medical College, especially Tanu Rana for their assistance with microscopy assays.

Conflicts of Interest: The authors declare no conflict of interest. The funders had no role in the design of the study; in the collection, analyses, or interpretation of data; in the writing of the manuscript, or in the decision to publish the results.

\section{Abbreviations}

$\begin{array}{ll}\text { DMEM } & \text { Dulbecco's modified eagle medium } \\ \text { FBS } & \text { Fetal bovine serum } \\ \text { MHEC } & \text { Mouse heart endothelia cells } \\ \text { SAV1 } & \text { Salvador homolog } 1 \\ \text { TAZ } & \text { Transcriptional coactivator with PDZ-binding motif } \\ \text { TCCRT } & \text { Trypanosoma cruzi calreticulin } \\ \text { TEAD } & \text { Transcriptional enhancer factor (TEF) family/TEA domain (TEAD) family } \\ \text { TSP-1KO } & \text { Thrombospondin-1 knock out } \\ \text { WT } & \text { Wild type } \\ \text { YAP } & \text { Yes-associated protein }\end{array}$

\section{References}

1. Bern, C.; Messenger, L.A.; Whitman, J.D.; Maguire, J.H. Chagas Disease in the United States: A Public Health Approach. Clin. Microbiol. Rev. 2019, 33, 1. [CrossRef] [PubMed]

2. Bonney, K.M.; Luthringer, D.J.; Kim, S.A.; Garg, N.J.; Engman, D.M. Pathology and Pathogenesis of Chagas Heart Disease. Annu. Rev. Pathol. Mech. Dis. 2019, 14, 421-447. [CrossRef]

3. Coura, J.R.; Vinas, P.A. Chagas disease: A new worldwide challenge. Nature 2010, 465, S6-S7. [CrossRef]

4. Gascon, J.; Bern, C.; Pinazo, M.J. Chagas disease in Spain, the United States and other non-endemic countries. Acta Trop. 2010, 115, 22-27. [CrossRef] [PubMed] 
5. Hotez, P.J.; Dumonteil, E.; Cravioto, M.B.; Bottazzi, M.E.; Tapia-Conyer, R.; Meymandi, S.; Karunakara, U.; Ribeiro, I.; Cohen, R.M.; Pécoul, B. An Unfolding Tragedy of Chagas Disease in North America. PLoS Negl. Trop. Dis. 2013, 7, e2300. [CrossRef] [PubMed]

6. Lynn, M.K.; Bossak, B.H.; Sandifer, P.A.; Watson, A.; Nolan, M.S. Contemporary autochthonous human Chagas disease in the USA. Acta Trop. 2020, 205, 105361. [CrossRef]

7. Bornstein, P. Diversity of function is inherent in matricellular proteins: An appraisal of thrombospondin 1. J. Cell Boil. 1995, 130, 503-506. [CrossRef]

8. Bornstein, P. Thrombospondins as matricellular modulators of cell function. J. Clin. Investig. 2001, 107, 929-934. [CrossRef]

9. Murphy-Ullrich, J.E. Thrombospondin 1 and Its Diverse Roles as a Regulator of Extracellular Matrix in Fibrotic Disease. J. Histochem. Cytochem. 2019, 67, 683-699. [CrossRef]

10. Murphy-Ullrich, J.E.; Sage, E.H. Revisiting the matricellular concept. Matrix Boil. 2014, 37, 1-14. [CrossRef]

11. Adams, J.C.; Lawler, J. The thrombospondins. Cold Spring Harb. Perspect. Biol. 2011, 3, a009712. [CrossRef] [PubMed]

12. Bornstein, P.; Agah, A.; Kyriakides, T.R. The role of thrombospondins 1 and 2 in the regulation of cell-matrix interactions, collagen fibril formation, and the response to injury. Int. J. Biochem. Cell Boil. 2004, 36, 1115-1125. [CrossRef] [PubMed]

13. Resovi, A.; Pinessi, D.; Chiorino, G.; Taraboletti, G. Current understanding of the thrombospondin-1 interactome. Matrix Boil. 2014, 37, 83-91. [CrossRef] [PubMed]

14. Sweetwyne, M.T.; Murphy-Ullrich, J.E. Thrombospondin1 in tissue repair and fibrosis: TGF- $\beta$-dependent and independent mechanisms. Matrix Boil. 2012, 31, 178-186. [CrossRef] [PubMed]

15. Tan, K.; Lawler, J. The interaction of Thrombospondins with extracellular matrix proteins. J. Cell Commun. Signal. 2009, 3, 177-187. [CrossRef] [PubMed]

16. Krishna, S.M.; Golledge, J. The role of thrombospondin-1 in cardiovascular health and pathology. Int. J. Cardiol. 2013, 168, 692-706. [CrossRef] [PubMed]

17. Simmons, K.J.; Nde, P.N.; Kleshchenko, Y.Y.; Lima, M.F.; Villalta, F. Stable RNA interference of host thrombospondin-1 blocks Trypanosoma cruziinfection. FEBS Lett. 2006, 580, 2365-2370. [CrossRef]

18. Johnson, C.A.; Kleshchenko, Y.Y.; Ikejiani, A.O.; Udoko, A.N.; Cardenas, T.C.; Pratap, S.; Duquette, M.A.; Lima, M.F.; Lawler, J.; Villalta, F.; et al. Thrombospondin-1 Interacts with Trypanosoma cruzi Surface Calreticulin to Enhance Cellular Infection. PLoS ONE 2012, 7, e40614. [CrossRef]

19. Suman, S.; Rachakonda, G.; Mandape, S.; Sakhare, S.S.; Villalta, F.; Pratap, S.; Lima, M.F.; Nde, P.N. Phospho-proteomic analysis of primary human colon epithelial cells during the early Trypanosoma cruzi infection phase. PLoS Negl. Trop. Dis. 2018, 12, e0006792. [CrossRef]

20. Pan, D. The Hippo Signaling Pathway in Development and Cancer. Dev. Cell 2010, 19, 491-505. [CrossRef]

21. Yu, F.-X.; Zhao, B.; Guan, K.-L. Hippo Pathway in Organ Size Control, Tissue Homeostasis, and Cancer. Cell 2015, 163, 811-828. [CrossRef] [PubMed]

22. Boopathy, G.T.K.; Hong, W. Role of Hippo Pathway-YAP/TAZ Signaling in Angiogenesis. Front. Cell Dev. Boil. 2019, 7, 49. [CrossRef] [PubMed]

23. Hansen, C.G.; Moroishi, T.; Guan, K.-L. YAP and TAZ: A nexus for Hippo signaling and beyond. Trends Cell Boil. 2015, 25, 499-513. [CrossRef] [PubMed]

24. Meng, Z.; Moroishi, T.; Guan, K.-L. Mechanisms of Hippo pathway regulation. Genes Dev. 2016, $30,1-17$. [CrossRef]

25. Shen, J.; Cao, B.; Wang, Y.; Ma, C.; Zeng, Z.; Liu, L.; Li, X.; Tao, D.; Gong, J.; Xie, D. Hippo component YAP promotes focal adhesion and tumour aggressiveness via transcriptionally activating THBS1/FAK signalling in breast cancer. J. Exp. Clin. Cancer Res. 2018, 37, 175. [CrossRef]

26. Nunes, M.C.P.; Beaton, A.; Acquatella, H.; Bern, C.; Bolger, A.F.; Echeverría, L.E.; Dutra, W.O.; Gascon, J.; Morillo, C.A.; Oliveira-Filho, J.; et al. Chagas Cardiomyopathy: An Update of Current Clinical Knowledge and Management: A Scientific Statement From the American Heart Association. Circulation 2018, 138, e169-e209. [CrossRef]

27. Ferreira, A.V.M.; Segatto, M.; Menezes, Z.; Macedo, A.M.; Gelape, C.; Andrade, L.D.O.; Nagajyothi, F.; Scherer, P.E.; Teixeira, M.M.; Tanowitz, H.B. Evidence for Trypanosoma cruzi in adipose tissue in human chronic Chagas disease. Microbes Infect. 2011, 13, 1002-1005. [CrossRef] 
28. Lewis, M.D.; Kelly, J.M. Putting Infection Dynamics at the Heart of Chagas Disease. Trends Parasitol. 2016, 32, 899-911. [CrossRef] [PubMed]

29. A Callus, B.; Verhagen, A.M.; Vaux, D.L. Association of mammalian sterile twenty kinases, Mst1 and Mst2, with hSalvador via C-terminal coiled-coil domains, leads to its stabilization and phosphorylation. FEBS J. 2006, 273, 4264-4276. [CrossRef]

30. Chan, E.H.Y.; Nousiainen, M.; Chalamalasetty, R.B.; Schäfer, A.; A Nigg, E.; Silljé, H.H.W. The Ste20-like kinase Mst2 activates the human large tumor suppressor kinase Lats1. Oncogene 2005, 24, 2076-2086. [CrossRef]

31. Zhou, D.; Conrad, C.; Xia, F.; Park, J.-S.; Payer, B.; Yin, Y.; Lauwers, G.Y.; Thasler, W.; Lee, J.T.; Avruch, J.; et al. Mst1 and Mst2 Maintain Hepatocyte Quiescence and Suppress Hepatocellular Carcinoma Development through Inactivation of the Yap1 Oncogene. Cancer Cell 2009, 16, 425-438. [CrossRef] [PubMed]

32. Hong, W.; Guan, K.-L. The YAP and TAZ transcription co-activators: Key downstream effectors of the mammalian Hippo pathway. Semin. Cell Dev. Boil. 2012, 23, 785-793. [CrossRef] [PubMed]

33. Luo, X. Snapshots of a hybrid transcription factor in the Hippo pathway. Protein Cell 2010, 1, 811-819. [CrossRef] [PubMed]

34. Nde, P.N.; Lima, M.F.; Johnson, C.A.; Pratap, S.; Villalta, F. Regulation and use of the extracellular matrix by Trypanosoma cruzi during early infection. Front. Immunol. 2012, 3, 337. [CrossRef] [PubMed]

35. De Araújo-Jorge, T.C.; Waghabi, M.C.; Soeiro, M.D.N.C.; Keramidas, M.; Bailly, S.; Feige, J.-J. Pivotal role for TGF- $\beta$ in infectious heart disease: The case of Trypanosoma cruzi infection and consequent Chagasic myocardiopathy. Cytokine Growth Factor Rev. 2008, 19, 405-413. [CrossRef]

36. Ferreira, R.R.; Abreu, R.D.S.; Vilar-Pereira, G.; Degrave, W.; Meuser-Batista, M.; Ferreira, N.V.C.; Da Cruz Moreira, O.; Da Silva Gomes, N.L.; Mello de Souza, E.; Ramos, I.P.; et al. TGF-beta inhibitor therapy decreases fibrosis and stimulates cardiac improvement in a pre-clinical study of chronic Chagas' heart disease. PLoS Negl. Trop. Dis. 2019, 13, e0007602. [CrossRef]

37. Hall, B.S.; Pereira, M.E.A. Dual Role for Transforming Growth Factor $\beta$-Dependent Signaling in Trypanosoma cruzi Infection of Mammalian Cells. Infect. Immun. 2000, 68, 2077-2081. [CrossRef]

38. Chen, Y.; Leask, A.; Abraham, D.J.; Kennedy, L.; Shi-Wen, X.; Denton, C.P.; Black, C.M.; Verjee, L.S.; Eastwood, M. Thrombospondin 1 is a key mediator of transforming growth factor beta-mediated cell contractility in systemic sclerosis via a mitogen-activated protein kinase kinase (MEK)/extracellular signal-regulated kinase (ERK)-dependent mechanism. Fibrogenesis Tissue Repair 2011, 4, 9. [CrossRef]

39. Murphy-Ullrich, J.E.; Suto, M.J. Thrombospondin-1 regulation of latent TGF-beta activation: A therapeutic target for fibrotic disease. Matrix Biol. 2018, 68, 28-43. [CrossRef]

40. Nor, J.E.; Dipietro, L.; Murphy-Ullrich, J.E.; Hynes, R.O.; Lawler, J.; Polverini, P.J. Activation of Latent TGF-beta1 by Thrombospondin-1 is a Major Component of Wound Repair. Oral Biosci. Med. 2005, 2, $153-161$.

41. Su, X.; Sorenson, C.M.; Sheibani, N. Isolation and characterization of murine retinal endothelial cells. Mol. Vis. 2003, 9, 171-178. [PubMed]

42. Lima, M.F.; Villalta, F. Trypanosoma cruzi trypomastigote clones differentially express a parasite cell adhesion molecule. Mol. Biochem. Parasitol. 1989, 33, 159-170. [CrossRef]

43. Udoko, A.N.; Johnson, C.A.; Dykan, A.; Rachakonda, G.; Villalta, F.; Mandape, S.; Lima, M.F.; Pratap, S.; Nde, P.N. Early Regulation of Profibrotic Genes in Primary Human Cardiac Myocytes by Trypanosoma cruzi. PLoS Negl. Trop. Dis. 2016, 10, e0003747. [CrossRef] [PubMed]

44. Villalta, F.; Lima, M.F.; Zhou, L. Purification of Trypanosoma cruzi surface proteins involved in adhesion to host cells. Biochem. Biophys. Res. Commun. 1990, 172, 925-931. [CrossRef]

(C) 2020 by the authors. Licensee MDPI, Basel, Switzerland. This article is an open access article distributed under the terms and conditions of the Creative Commons Attribution (CC BY) license (http://creativecommons.org/licenses/by/4.0/). 